Check for updates

Cite this: RSC Adv., 2018, 8, 20809

\title{
Biodistribution and targeting potential assessment of mucoadhesive chitosan nanoparticles designed for ulcerative colitis via scintigraphy $\dagger$
}

\begin{abstract}
Pooja Mongia Raj, ${ }^{a}$ Rakesh Raj, (D) ankur Kaul, ${ }^{b}$ Anil K. Mishra ${ }^{b}$ and Alpana Ram ${ }^{a}$
In the present investigation we have prepared and characterized curcumin (CN)-containing chitosan nanoparticles (CS-NPs) coated with Eudragit FS 30D for colon-specific drug delivery for treatment of ulcerative colitis. Methods: CS-NPs were prepared by ionic gelation using tripolyphosphate. To specify $\mathrm{pH}$ sensitive delivery, CS-CN-NPs were coated with Eudragit FS 30D by using a solvent evaporation method. Different process parameters were evaluated, and the optimized formulation was characterized by particle size, size distribution, zeta potential and encapsulation efficiency before lyophilization. The lyophilized product was further subjected to Fourier-transform infrared spectroscopy, and particle morphology and in vitro drug release in different media were studied. Results: the kinetics of in vitro drug release from the CS-CN-NPs revealed sustained release behaviour of the developed carriers. In vivo biodistribution study by gamma-scintigraphy showed good accumulation of the developed nanocarriers in the colonic region. Conclusion: sustained and $\mathrm{pH}$ stimulated delivery of $\mathrm{CN}$ to the colon was successfully attained via coating of CS-NPs with Eudragit FS 30D to circumvent poor absorption and availability of $\mathrm{CN}$.
\end{abstract}

Received 20th March 2018

Accepted 8th May 2018

DOI: $10.1039 / \mathrm{c} 8 \mathrm{ra01898g}$

rsc.li/rsc-advances

Application of anti-inflammatory or immunosuppressive

\section{Introduction}

Ulcerative colitis (UC) and Crohn's disease (CD) are described as inflammatory bowel disease (IBD). IBD is a set of pathological conditions that lead to chronic inflammation and impairment of the gastrointestinal tract (GIT). ${ }^{1,2}$ They are idiopathic and both are evident as persistent inflammation of the GIT, but they differ in the nature and site of inflammation. In the case of UC, colon and rectum are affected, limited to mucosa and continuous inflamed areas (which are a characteristic feature), whereas any of the parts of the GIT prone to be affected in CD are transmural and are characterized by segmental inflamed areas., Abnormal interaction of gastrointestinal microflora with the mucosal immune system is commonly observed in UC patients. ${ }^{5}$ The treatments employed for the management of UC include aminosalicylates, corticosteroids and their derivatives, immunomodulators, and several other therapies, including integrin antibody therapy, recombinant growth factors, macrolide combination antibody therapy, monoclonal antibody, and mitogen-activated protein kinase inhibitors. The key goal of UC treatment is to reduce inflammation with anti-inflammatory drugs.

${ }^{a}$ Institute of Pharmaceutical Sciences, Guru Ghasidas Vishwavidyalaya, Bilaspur, C.G. 495009, India. E-mail: rajscientist.2014@rediffmail.com; Tel: +917752-260027

${ }^{b}$ Division of Cyclotron and Radiopharmaceutical Sciences, Institute of Nuclear Medicine and Allied Sciences (INMAS), New Delhi 110054, India

$\dagger$ Electronic supplementary information (ESI) available. See DOI: $10.1039 / \mathrm{c} 8 \mathrm{ra} 01898 \mathrm{~g}$ agents is very frequently associated with various common toxic effects related to dose (vomiting, headache, malaise, haemolysis and nausea) or idiosyncratic (fever, rash, lymphadenopathy, agranulocytosis and pneumonitis) and antibiotics intolerances. ${ }^{6,7}$ No complete remission can be accomplished by means of the above-mentioned treatments and repeated flares of inflammation are experienced by most patients, who also develop resistance to therapy. Patients with the chronic active disease experience destruction and loss of the epithelial surface of the mucosa as a result of repeated or continuous inflammation. This causes enhanced proliferation of epithelial cells which when uncontrolled results in colitis-associated colorectal cancers. $^{\mathbf{8 , 9}}$

Recently, various studies have focused on the treatment of inflammatory disease with a natural moiety to avoid the risk of adverse effects. A number of such reports show that curcumin (CN) can attenuate inflammation in a mouse colitis model and clinically minimize the rate of UC relapse. ${ }^{\mathbf{1 0 , 1 1}} \mathrm{CN}$, a hydrophobic phenol derivative obtained from Curcuma longa Linn, possesses numerous therapeutic activities, such as anti-cancer, antioxidant, antimicrobial along with anti-inflammatory activity, and is gaining higher interest for UC therapy. It promotes mucosal healing by significantly downregulating the inflammatory cytokines and scavenges free radicals. ${ }^{12,13}$ Venkataraganna and others have reported that $\mathrm{CN}$ has pronounced inhibitory effect on inducible nitric oxide synthase (iNOS) and nuclear factor kappa B (NF-кB). ${ }^{\mathbf{1 1 , 1 4 , 1 5}}$ The U.S. Food and Drug 
Administration (FDA) has approved curcumin as a GRAS (Generally Recognized As Safe) drug since various clinical trials revealed that even at a high dose $\left(12 \mathrm{~g}\right.$ day $\left.^{-1}\right)$ for 3 months curcumin is harmless for humans. ${ }^{\mathbf{1 6 , 1 7}}$ However, the major drawback of curcumin is low bioavailability via the oral route due to its very low aqueous solubility, ${ }^{18}$ and short half-life because of high metabolic rate. ${ }^{\mathbf{1 9 2 0}}$ To overcome these limitations, various novel sustained delivery systems have been reported for specifying curcumin availability directly to colitis tissues, such as tablets, liposomes, microspheres, nanostructured lipid carriers, self-emulsifying drug delivery system (SEDDS) and pellets. ${ }^{10,21-23}$ However, these suffer from wellknown limitations, including unintentional release throughout the GIT, long term stability and lower penetration through the mucus layer. Such obstacles can be overcome by nanoparticles (NPs). For example, solid lipid nanoparticles ${ }^{24}$ and chitosan-montmorillonite clay NPs were reported as sustained delivery carriers for curcumin, but showed drug release throughout the GIT. ${ }^{25,26}$ As already reported, NPs are taken up through macrophages in the active inflamed area of UC, and adherence of small particles on the thicker mucus layer (high mucus production in active UC) in the inflamed region is also high. The smaller size of NPs makes them capable of accumulating in the inflamed area. ${ }^{27}$

NPs made from biodegradable materials like chitosan (CS), pectin, alginate, gelatin, gums etc. have gained much attention. Among these, chitosan is a very good example of a biodegradable polymer widely employed for development of NPs. ${ }^{28,29}$ It is mucoadhesive, which prolongs the contact time of drugs with biological membranes and modifies the permeability of the mucosal surface; owing to this specific characteristic, it has been gaining more interest in the last decade. ${ }^{30,31}$ Chitosan is a cationic polysaccharide and can interact with negatively charged mucosal surface. ${ }^{32}$ Though having many advantages, the use of chitosan in delivery to the colon is limited owing to its degradation at acidic $\mathrm{pH}$ and interaction with the mucosal surface of the entire GIT. Hence most studies have reported on administration of CN encapsulated in CS-NPs via the parenteral route to provide sustained delivery of drug for breast cancer, hepatocarcinoma and tumour cells at pH 5 or above. ${ }^{33-36}$ Some reports on CS-CN-NPs revealed enhanced bioavailability of anticancer and antimalarial drugs in comparison with pure $\mathrm{CN}$ but did not show the effect of acidic $\mathrm{pH}$ on release of NPs. ${ }^{37-39}$ In this study, the coating of chitosan nanoparticles with $\mathrm{pH}$ sensitive Eudragit polymer was exhaustively studied to overcome the effect of highly acidic environment. ${ }^{19,40,41}$

Eudragit FS 30D is a pH sensitive polymer that prevents the release of drug in the stomach and proximal small intestine. ${ }^{42}$ It is an anionic copolymer of methyl acrylate, methylmethacrylate and methacrylic acid. Eudragit FS 30D dissolves similarly to Eudragit $\mathrm{S} 100$ at $\mathrm{pH} 7$, but in a more sustained and controlled mode. ${ }^{43}$ Since the $\mathrm{pH}$ at the ileum and the ileo-caecal valve is reported to be $7-8$, it is expected that Eudragit FS $30 \mathrm{D}$ will dissolve in that region. ${ }^{\mathbf{4 4}}$

The present investigation focused on developing and characterizing chitosan based mucoadhesive NPs loaded with $\mathrm{CN}$ by an ionic gelation method and coated with $\mathrm{pH}$ triggered polymer. The further optimized formulation was subjected to studies of in vitro drug release kinetics, biodistribution and targeting potential by scintigraphy.

\section{Result and discussion}

UC drastically affects the quality of a patient's life and treatment carries a cost burden. Along with this, patients are also at a greater risk of colon cancer and liver disease. There is no standard treatment protocol available for managing all patients with UC. The approach to treat UC must be adapted to an individual; treatment is based on aminosalicylates, corticosteroids, immunomodulators, antibiotics and monoclonal antibodies, and every therapy has adverse effects. Curcumin is a herbal compound that can reduce UC and alleviate the inflammatory response. Several studies have demonstrated the role of curcumin as a novel therapy for children and adults with $\mathrm{UC}$; the mode of action of curcumin in the treatment of UC is unknown, but abundant evidence has established its effects on the NF- $\kappa$ B pathway and p38 MAPK in the intestinal mucosa. ${ }^{45}$ We have developed CN-NPs using low molecular weight CS with a high degree of deacetylation ( $>95 \%$ ) because it is soluble in low acid media. Besides this, more amino groups are available, which could facilitate the electrostatic interaction of CS with tripolyphosphate (TPP). Smaller particle size and narrow size distribution of highly deacetylated CS has already been reported in the previous literature. ${ }^{46}$

Eudragit FS 30D coated CS-NPs encapsulating the herbal drug CN (FS-CS-CN-NPs) were prepared by an ionic gelation method as shown schematically in Fig. 1. The ionic gelation method makes particles in the nano-size range with low polydispersity index and appropriate zeta potential. An adequate charge density is needed for anions to cross-link CS and form particles. When the concentration of CS was varied from 0.05 to $0.3 \%(\mathrm{w} / \mathrm{v})$ and the volume ratio of CS : TPP was $2: 1,2.5: 1$, $3: 1,4: 1$ and $5: 1$, the size of the NPs was found to be 161$439 \mathrm{~nm}$, with polydispersity index less than 1 and zeta potential from 22 to $30 \mathrm{mV}$, as shown in Table 1. Positive charge is suitable for uptake of NPs in the inflamed colonic mucosa. Zeta potential also affects the intracellular localization and mucoadhesive property of NPs. The greater localization of CS$\mathrm{CN}-\mathrm{NPs}$ to the inflamed colon has the advantages of higher drug concentration and lower drug degradation.

The size of NPs was found to increase with an increase in CS concentration, which also affects the entrapment efficiency (EE). For the different formulations used during the process of optimization, EE varied from $70.53 \%$ to $89.51 \%$. The EE was found to decrease upon increasing CS : TPP ratio from $4: 1$ to $5: 1$, possibly due to inefficient cross-linking taking place at higher CS contents. A formulation consisting of drug:polymer in $1: 10$ ratio and CS-TPP in $3: 1$ ratio exhibited the highest EE. We hypothesized that $\mathrm{CN}$ in the coated CS-CN-NPs was preserved as it passed through the upper GIT region, and then localized in the inflamed area after solubilization of the FS 30D coating. The Eudragit FS 30D coating was applied on CS-NPs by oil-in-oil emulsification solvent evaporation technique using 


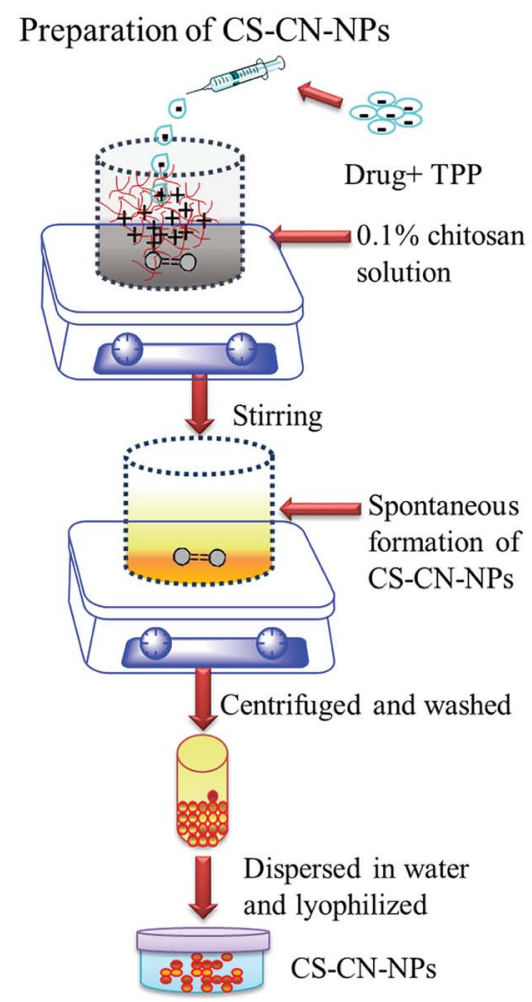

Coating of CS-CN-NPs with Eudragit

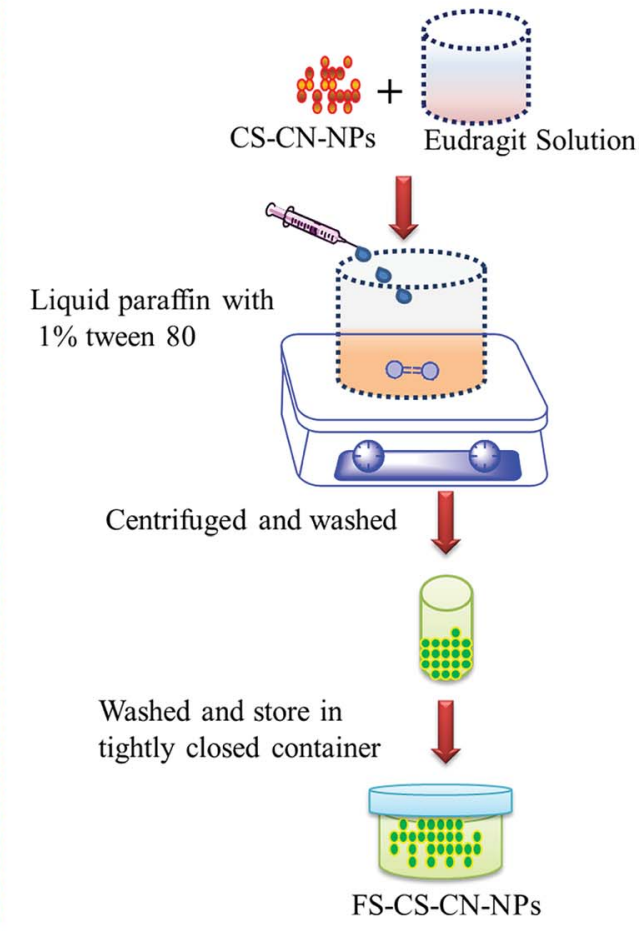

Fig. 1 Schematic representation of preparation of chitosan nanoparticles and coating with Eudragit FS 30D.

a coat : core ratio of $3: 1$. The coating of optimized CS-NPs by Eudragit FS 30D showed increases in size up to $368 \pm 3.9 \mathrm{~nm}$.

In accordance with the ICH guidelines the developed HPLC method was validated for precision, accuracy, linearity, limit of detection (LOD) and limit of quantification (LOQ), as shown in Table 2. The correlation coefficient $\left(r^{2}\right)$ of $\mathrm{CN}$ was found to be 0.999 , with linearity in the range of $5-50 \mu \mathrm{g} \mathrm{ml}^{-1}$. The limit of detection (LOD) for CN was $3.370 \mu \mathrm{g} \mathrm{ml}^{-1}$ at a wavelength of $425 \mathrm{~nm}$. Suitability of the method was tested by determining percentage relative standard deviations (\% RSD) of peak area and retention time, which were found to be less than $2 \%$. By using the signal to noise ratio 10 , the limit of quantitation (LOQ) of CN was detected. The percentage recovery of the $\mathrm{CN}$ from the developed method was well within $100.19 \pm 1.93 \%$, and \%RSD of the assay of intra-day and inter-day precision was less than $2 \%$, as depicted in Table 3.

The water uptake and swelling rate of polymer play an important role in drug release profile; this may affect the rate of drug absorption at the site of delivery and cellular uptake. In this study, in vitro swelling behaviour of the prepared carriers revealed the percentage swelling in phosphate buffer $\mathrm{pH}$ 1.2, 6.8 and 7.4 was $474 \pm 24 \%, 196 \pm 17 \%$ and $152 \pm 17 \%$ respectively in $24 \mathrm{~h}$. The $\mathrm{pH}$ dependent swelling behaviour of CS-NPs is due to its higher solubility in acidic media and the creation of pores. This phenomenon leads to diffusion of the drug from the polymer surface and is responsible for the initial burst release from CS-CN-NPs. The swelling of CS-NPs increased with time, but maximum swelling occurred after $4 \mathrm{~h}$; after that slight swelling was observed (Fig. 2).

\section{Fourier-transform infrared spectrometry (FTIR)}

FTIR spectra confirmed the formation of chitosan nanoparticles by efficient cross-linking with sodium tripolyphosphate. Spectra of chitosan powder, curcumin, Eudragit FS 30D, CS-NPs, CSCN-NPs and FS-CS-CN-NPs are shown in Fig. 3.

Usually, chitosan powder shows distinctive peaks at 35353003 ( $-\mathrm{OH}$ and $-\mathrm{NH}_{2}$ stretching), 2877 ( $-\mathrm{CH}$ stretching), 1658 (amide I), 1259 (C-O-C stretching) and $1728 \mathrm{~cm}^{-1}$ (carbonyl group) (Fig. 3a). The spectrum of $\mathrm{CN}$ (Fig. 3b) shows characteristic absorption bands at 3510 to 3089 (phenolic OH), at 1627 (the $\mathrm{C}=\mathrm{O}$ stretching peak of conjugate ketone), at 1512 and

Table 1 Characterization of CS-NPs, CS-CN-NPs and FS-CS-CN-NPs (mean \pm SD, $n=3$ )

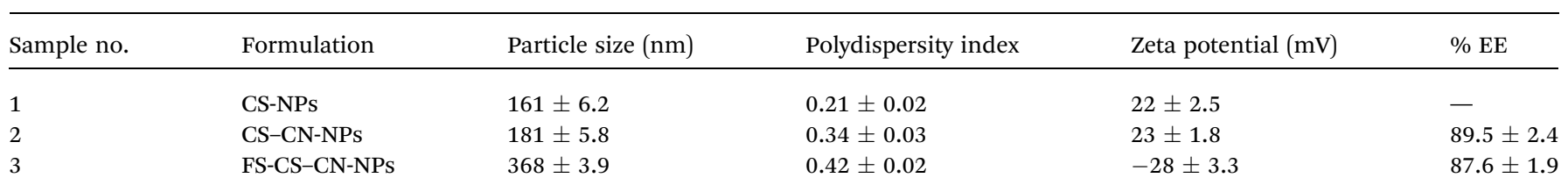


Table 2 Summary of HPLC validation parameters of curcumin

\begin{tabular}{ll}
\hline Parameter & Value \\
\hline Accuracy & $100.199 \pm 1.930$ \\
Slope & 12.73 \\
Intercept & 13.705 \\
Linearity range & $5-50 \mu \mathrm{g} \mathrm{ml}^{-1}$ \\
Correlation coefficient & 0.999 \\
SE of intercept & 4.596 \\
SD of intercept & 13.00 \\
LOD & 3.370 \\
LOQ & 10.212
\end{tabular}

1429 (aromatic and aliphatic $\mathrm{C}=\mathrm{C}$ stretching respectively) and the C-O-C stretching peak of ether at 1031. In CH-NPs (Fig. 3), the peak due to amide I ( $-\mathrm{NH}_{2}$ bending) shifted from 1658 to $1672 \mathrm{~cm}^{-1}$ and $1546 \mathrm{~cm}^{-1}$ (amide II), implying complex formation via electrostatic interaction between $\mathrm{NH}_{3}{ }^{+}$groups of chitosan and phosphoric groups of TPP within the nanoparticles. Two ionic interactions exist in the reaction process: an intense electrostatic interaction between the positively charged amino group of chitosan and the negative anion of TPP. The spectrum of FS 30D in Fig. 3d shows a characteristic band due to $\mathrm{C}=\mathrm{O}$ (vibrations of esterified carboxyl groups) at $1735 \mathrm{~cm}^{-1}$, which overlaps the $\mathrm{C}=\mathrm{O}$ band (vibrations of the carboxylic acid groups); further ester vibrations are detected at 1165, 1195, $1230,1253 \mathrm{~cm}^{-1}$. The wide absorption range of associated $-\mathrm{OH}$ groups between 3541 to 3072 is superimposed by $-\mathrm{CH}$ vibrations at $2951 \mathrm{~cm}^{-1}$ and further-CH vibrations can be discerned at 1382 and $1438 \mathrm{~cm}^{-1}$. This ionic interactions attest notably to the formation of CS-CN-NPs by cross-linking.

\section{Morphology of NPs through SEM and TEM}

TEM imaging of CS-CN-NPs and FS-CS-CN-NPs shows a uniform spherical structure with slight variation (Fig. 4A and B). The SEM micrograph (Fig. 5A and B) of CS-CN-NPs shows slightly rough surface, while spherical shape with smooth surface was observed after Eudragit coating of CS-CN-NPs.

Table 3 Intra- and inter-day precision for curcumin

\begin{tabular}{lccc}
\hline \multicolumn{5}{c}{$\begin{array}{c}\text { Concentration found, } \\
\text { Concentration }\left(\mu \mathrm{g} \mathrm{m}^{-1}\right)\end{array}$} & & \\
\hline Intra-day variation $(\boldsymbol{n}=\mathbf{5}$ at each level $)$ & & \\
5 & $4.91 \pm 0.06$ & 1 & \\
25 & $26.23 \pm 0.41$ & 1 & 1.22 \\
50 & $51.10 \pm 0.38$ & 1 & 0.74 \\
5 & $5.02 \pm 0.06$ & 2 & 1.19 \\
25 & $26.17 \pm 0.51$ & 2 & 1.94 \\
50 & $50.86 \pm 0.43$ & 2 & 0.84 \\
5 & $4.88 \pm 0.08$ & 3 & 1.63 \\
25 & $25.06 \pm 0.38$ & 3 & 1.51 \\
50 & $50.31 \pm 0.52$ & 3 & 1.03
\end{tabular}

Inter-day variation ( $n=15$ each day, $n=3$ at each level)

\begin{tabular}{|c|c|c|}
\hline 5 & $4.86 \pm 0.07$ & - \\
\hline 25 & $25.23 \pm 0.48$ & - \\
\hline 50 & $50.05 \pm 0.35$ & - \\
\hline
\end{tabular}

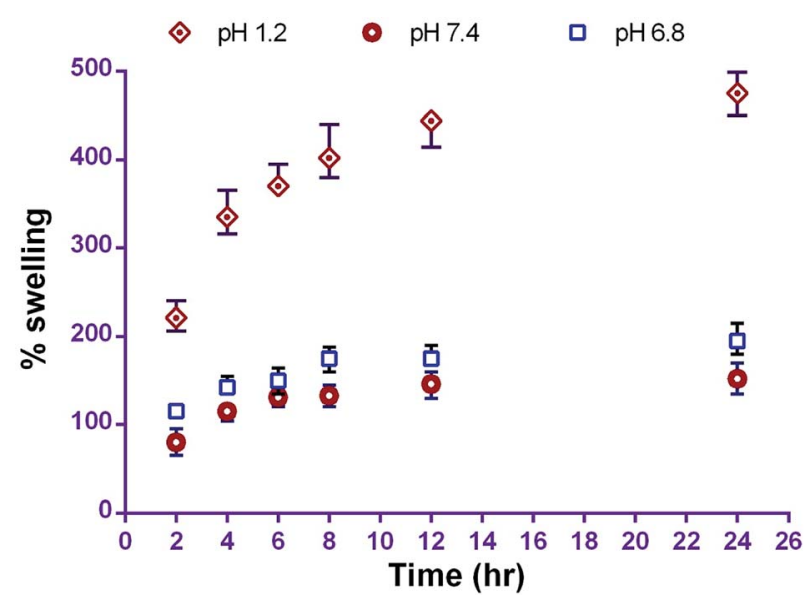

Fig. 2 Percentage swelling of CS-NPs at different $\mathrm{pH}$ (mean $\pm \mathrm{SD}, n=3$ ).

\section{In vitro release study}

The drug release behaviour from CS-CN-NPs and FS-CS-CNNPs was observed (Fig. 6) using a dialysis bag method. The release of curcumin from CS-CN-NPs and FS-CS-CN-NPs in different dissolution media was analysed through HPLC as described previously. An initial burst release of $\mathrm{CN}$ from uncoated NPs and then comparatively controlled release were observed. The initial burst release of drug from CS-CN-NPs may occur due to the immense swelling of CS, which is responsible for pore formation in the polymer surface and diffusion of the adsorbed drug from the carriers. In simulated gastric fluid (SGF) (at $\mathrm{pH}$ 1.2) the $\mathrm{CN}$ release from CS-CN-NPs started immediately on contact with the acidic condition and up to 54.3 $\pm 3 \%$ was released in $6 \mathrm{~h}$ (at $\mathrm{pH}$ 6.8). No release of $\mathrm{CN}$ from the Eudragit coated formulation (FS-CS-CN-NPs) at $\mathrm{pH} 1.2, \mathrm{pH} 4.5$ and $\mathrm{pH} 6.8$ was observed. This significant difference $(p<0.5)$ could be due to the coating of the formulation with $\mathrm{pH}$ dependent polymer that retards solublization of chitosan. This indicated that, owing to lack of drug release within the upper GIT, the developed carriers were more suitable than dextran sulphate chitosan nanoparticles and surface modified gelatin nanoparticles. ${ }^{47,48}$ The reason behind this could be the presence of the carboxyl group of Eudragit FS30D which ionizes in neutral and alkaline media. At alkaline $\mathrm{pH}$, the integrity of the coating is disturbed, and $\mathrm{CN}$ release was observed in our experiments.

Further experiments revealed that up to $97 \pm 1.7 \%$ of $\mathrm{CN}$ was released from CS-CN-NPs in simulated colonic fluid (SCF) $\mathrm{pH}$ 7.4 within $24 \mathrm{~h}$, but the release of CN from FS-CS-CN-NPs started from $6 \mathrm{~h}(23 \pm 2 \%)$ and proceeded continuously in a controlled manner. The cumulative release of drug during 6$8 \mathrm{~h}$ from coated nanoparticles was $25.3 \pm 1.8 \%$ in SCF and $28 \pm$ $2.1 \%$ in SCF with rat caecal contents, while uncoated nanoparticles released only $13 \pm 2.5 \%$ drug in the presence of SCF at $\mathrm{pH}$ 7.4. Such behaviour indicated that more $\mathrm{CN}$ would be available in the colon from FS-CS-CN-NPs than from CS-CNNPs. 


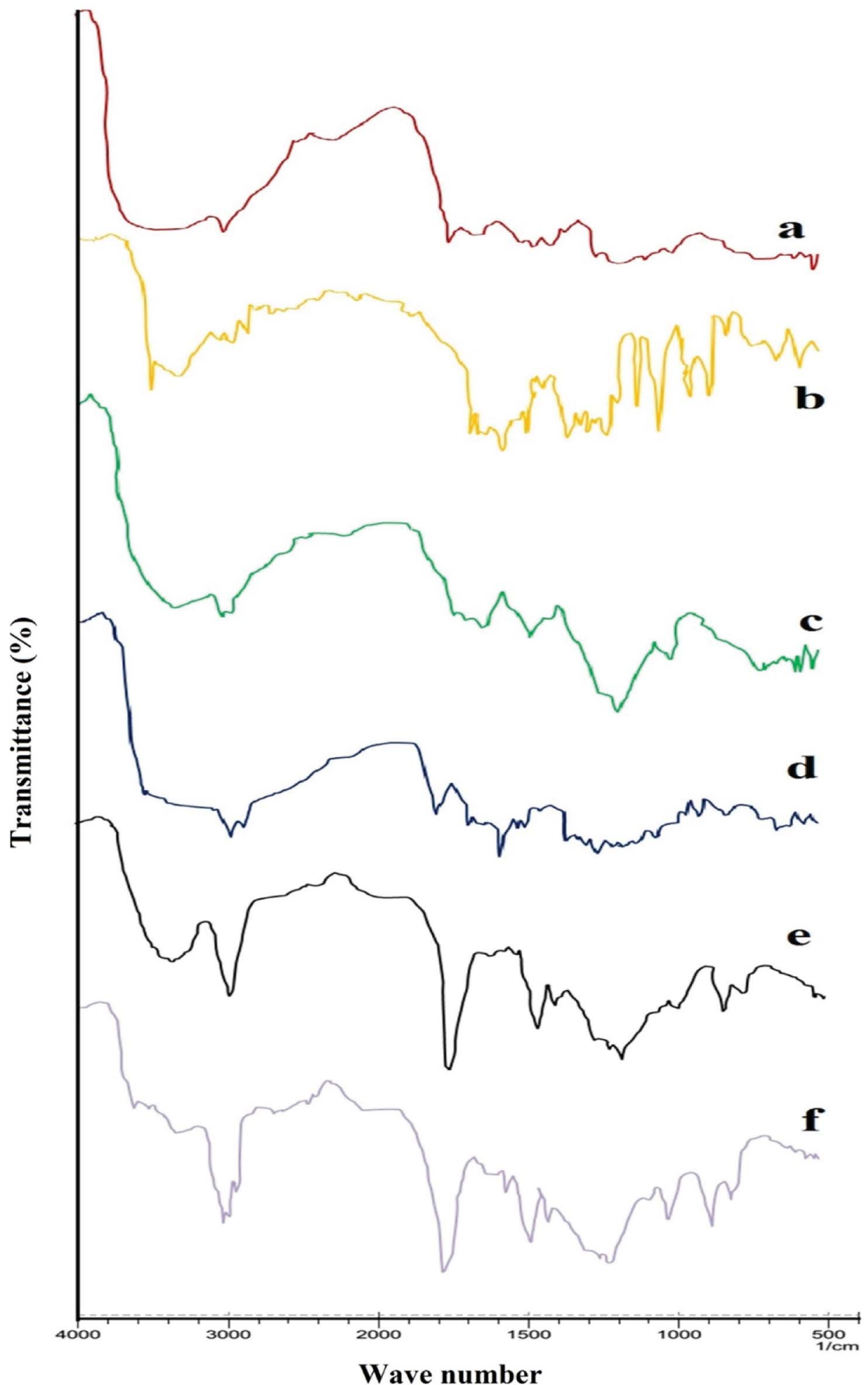

Fig. 3 FTIR spectra of (a) chitosan powder, (b) curcumin powder, (c) chitosan nanoparticles, (d) curcumin loaded chitosan nanoparticles, (e) Eudragit FS 30D and (f) Eudragit FS 30D coated chitosan nanoparticles.

Various studies reported CN release from modified chitosan NPs at pH 7.4 for up to 3-4 days in phosphate buffered saline without any gastric content. Such a system may be very attractive for parenteral delivery, ${ }^{49,50}$ but our goal is not only to avoid drug release in the upper GIT but also to achieve directed release of the therapeutic moiety in the colon by enzymatic degradation. Therefore, the in vitro release of FS-CS-CN-NPs was also determined in $\mathrm{pH} 7.4$ SCF containing $5 \% \mathrm{w} / \mathrm{v}$ rat caecal contents. With rat caecal contents, approximately $84.7 \pm$ $3 \%$ of the drug was released from FS-CS-CN-NPs in the colon 

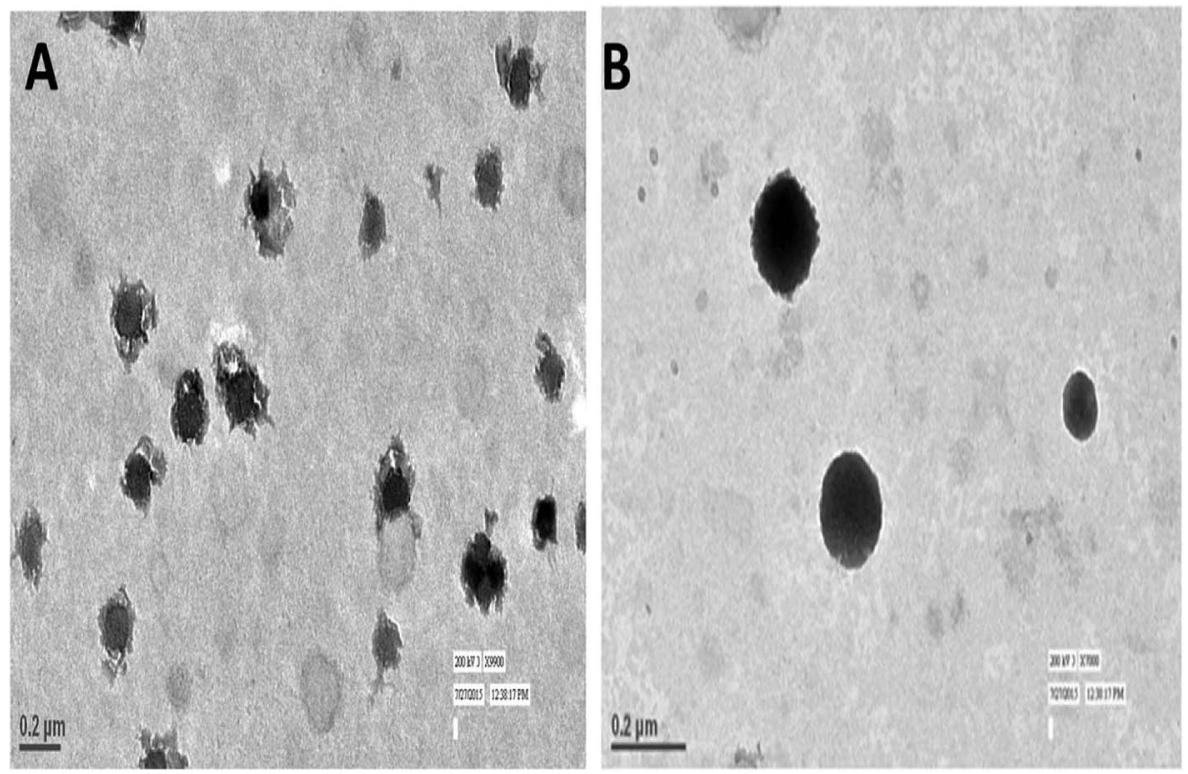

Fig. 4 TEM images of (A) CS-CN-NPs and (B) FS-CS-CN-NPs.

after protecting against release in the upper GIT, and $71.3 \pm 2 \%$ was released in the absence of rat caecal contents. Such sustained release behaviour in the colon could be more beneficial for maintaining therapeutic effects than blended NPs of chitosan. ${ }^{51}$ The rate of drug release from Eudragit coated CS-CN-NPs was higher in SCF containing rat caecal contents than without rat caecal content in the medium; this could be due to the presence of the enzyme beta-D-glucosaminidase, which acts on the $\beta-(1 \rightarrow 4)$ linkage of chitosan. ${ }^{52}$

\section{Release kinetics}

The release data of $\mathrm{CN}$ from the developed formulations were fitted into various mathematical models as shown in Table 4. The CN release profile from NPs (CS-CN-NPs and FS-CS-CNNPs) indicated that the Higuchi model is best suited for the release kinetics of the drug from both coated and uncoated formulations. The release exponent values for the sustained release of CN from CS-CN-NPs and FS-CS-CN-NPs are 0.519 and 1.098 respectively, which lie within the limits of the power law. The release exponent is a clear indication that anomalous diffusion (non-Fickian model) could be the principal release mechanism for $\mathrm{CN}$ from CS-CN-NPs, which is due to the combined effect of diffusion (due to swelling) and erosion for drug release, and super case II diffusion kinetics indicating swelling and relaxation of the polymer..$^{53}$

\section{Mucoadhesive study}

Bioadhesive CS-CN-NPs were fabricated and evaluated for mucoadhesive potential to confirm their higher local concentration by increasing retention time of curcumin at the inflamed region. As already reported, CS could interact with mucus glycoproteins by forming physical masses followed by
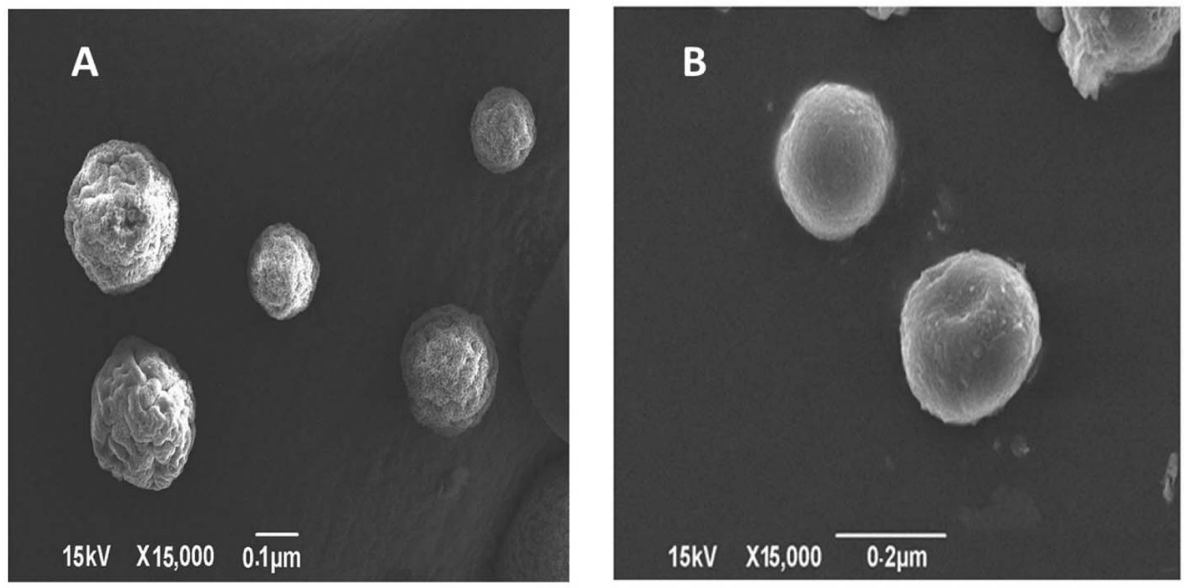

Fig. 5 SEM images of (A) CS-CN-NPs and (B) FS-CS-CN-NPS. 


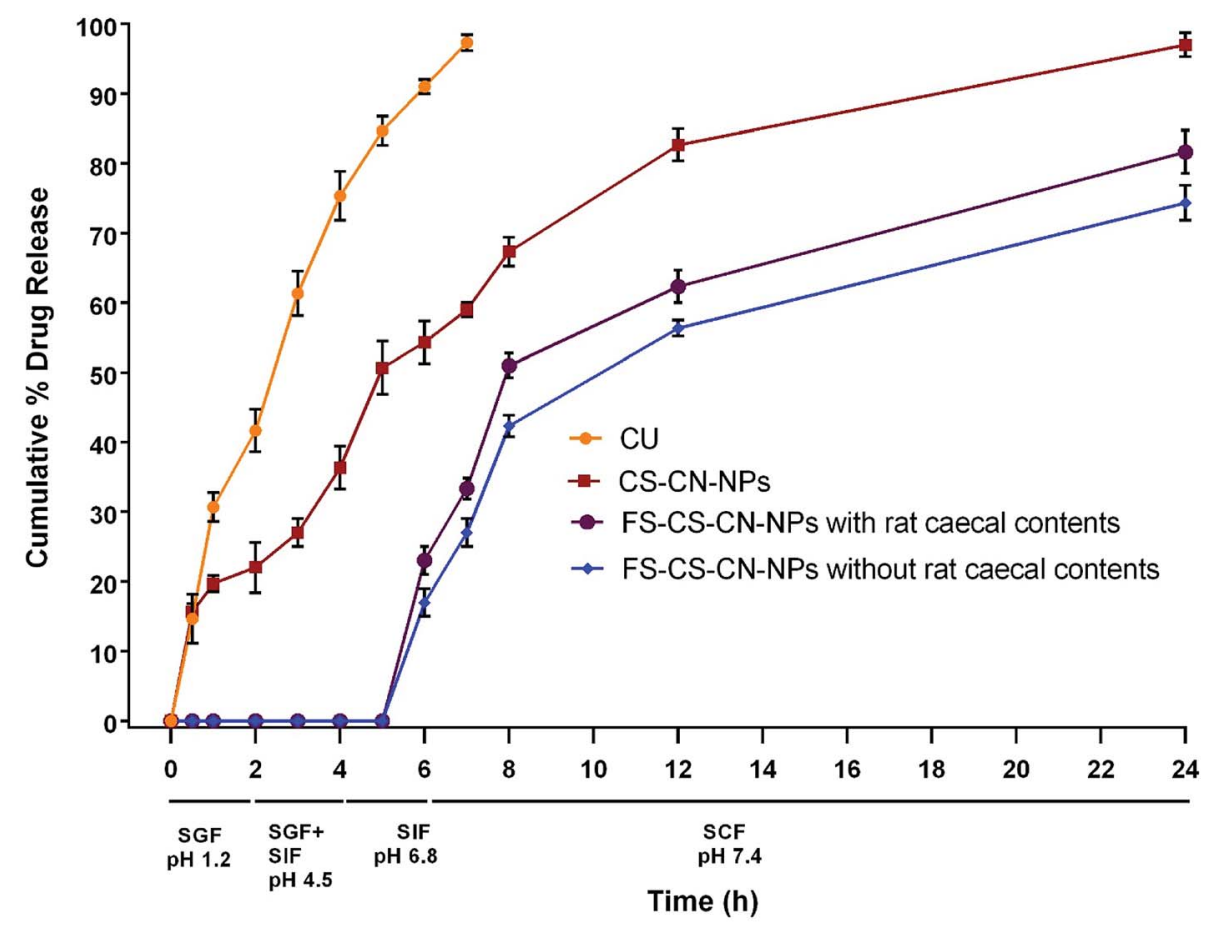

Fig. 6 Cumulative percentage release profile of curcumin (mean $\pm \mathrm{SD}, n=3$ ).

Table 4 Release kinetics for dissolution data of different formulations (mean $\pm \mathrm{SD}, n=3$ )

\begin{tabular}{llllll}
\hline & \multicolumn{2}{l}{ Correlation coefficient $r^{2}$ value } & & & \\
\cline { 2 - 6 } Formulation code & Zero order & First order & Higuchi model & Korsmeyer-Peppas model & Release exponent \\
\hline CS-CN-NPs & 0.821 & 0.991 & 0.992 & 0.976 & 0.519 \\
FS-CS-CN-NPs & 0.822 & 0.958 & 0.987 & 0.685 & 1.098 \\
\hline
\end{tabular}

hydrogen bonding with sugar residues on the oligosaccharides chains, and subsequently formation of a strengthened mucus gel complex, which permits the mucoadhesive materials to adhere for a prolonged period. ${ }^{54}$ The mucoadhesive properties of CS are also due to the electrostatic attraction of a negatively charged sialic acid group of mucin on intestinal epithelia and positive charge of the amino group present in CS. These characteristics of CS offer an extended contact time of the mucus layer surface with the polysaccharide based system to increase the localization and absorption in the targeted region to improve and increase the bioavailability of the drug molecules.

In this study, the mucoadhesive properties of CS-NPs and CS-CN-NPs were evaluated by measuring the amount of porcine stomach mucin (PSM) bound on NPs. In Fig. 7 the PSM (\% weight) bound to CS-NPs shows that the NPs without CN had the slightly higher mucoadhesion. There is no significant difference between the two ( $p$-value $>0.05)$ indicated by paired $t$ test. In the case of CS-CN-NPs, the amount of CS available for interacting with PSM decreases, leading to the lower mucoadhesive strength of NPs. Another factor which may contribute to the reduction of mucoadhesive strength of drug loaded NPs is an increase in NP size, which would decrease absorption of PSM on the surface of NPs (as the increase in size reduces the specific surface area of the NPs), leading to a decrease of the mucoadhesive strength of NPs. These results also correlate with

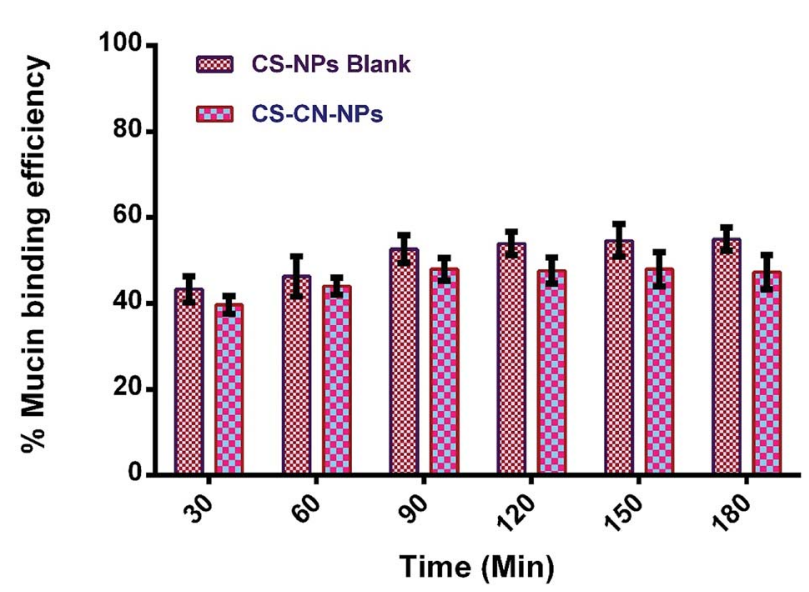

Fig. 7 Mucoadhesive study of (a) CS-NPs and (b) CS-CN-NPs (mean $\pm \mathrm{SD}, n=3)$. 
previously reported findings. ${ }^{55,56}$ The mucoadhesion strength of NPs progressively increased with time up to $90 \mathrm{~min}$, after which equilibrium for PSM interaction was reached.

\section{In vivo study}

All animal procedures were performed in accordance with the guidelines of the Committee for the Purpose of Control and Supervision of Experiments on Animals (CPCSEA), Government of India and approved by the Institutional Animal Ethical Committee of Guru Ghasidas University Bilaspur (994/GO/Ere/ S/06/CPCSEA).

\section{Gamma-scintigraphic evaluation}

The radiolabelling efficacy was determined by instant thin-layer chromatography (ITLC) using acetone as the mobile phase. The amount of stannous chloride (reducing agent) plays a very significant role in determining the extent of labelling. The stability of radiolabelled NPs was evaluated in normal saline and plasma. Fig. 8 shows the radiolabelled formulation was stable over $24 \mathrm{~h}$ and suitable for biodistribution and scintigraphy imaging. The delivery of NPs to different gastrointestinal organs was confirmed by the gamma-scintigraphy. Fig. 9 indicates that the FS-CS-NPs were localized in the intestinal region after $3 \mathrm{~h}$, which indicates the passage of NPs through the stomach to the intestine. After $8 \mathrm{~h}$, 'hot-spots' were detected in the large intestinal area. This study revealed good colon-specific targeting by the developed Eudragit coated nanoparticle formulation.

The organ distribution of labelled NPs was evaluated in different organs and tissues (heart, liver, lungs, kidney, spleen, stomach, intestine and colon), and fluids (blood and urine) and

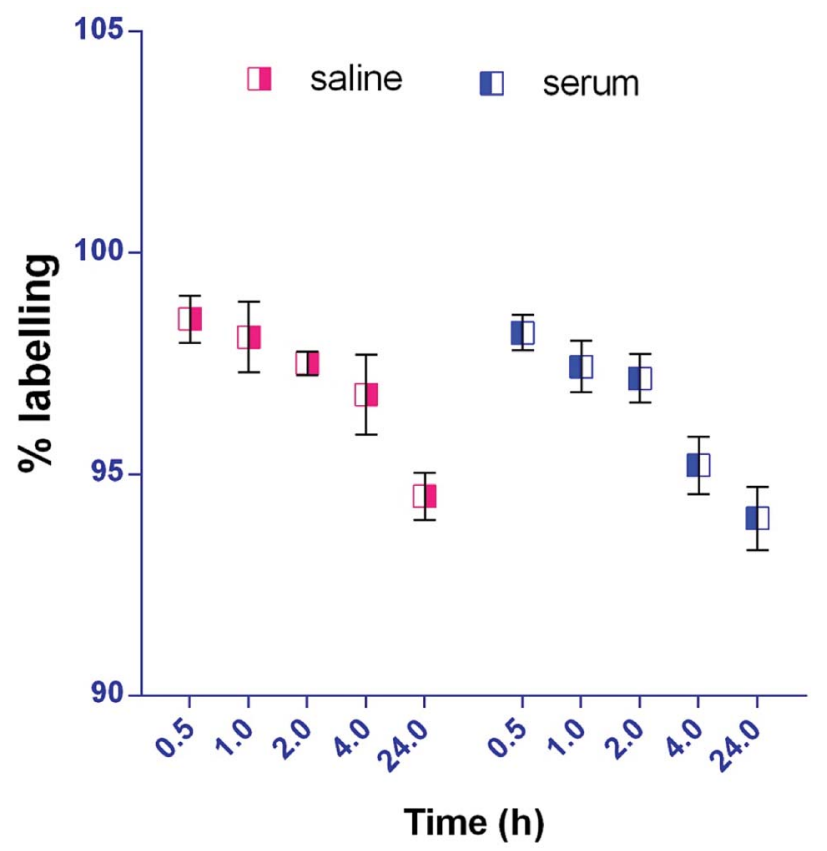

Fig. 8 In-vitro stability study of radiolabelled NPs in saline and serum (mean $\pm \mathrm{SD}, n=3$ ). is depicted in Fig. 10. The scintigraphic scan represents the localization of the radioactivity in the organs after $24 \mathrm{~h}$. The maximum concentration was found in the colon, followed by small-intestine, and only traces were found in the stomach; showing that the highest retention time of NPs was in the colon for local effect.

\section{Materials and methods}

\section{Materials}

Chitosan (low molecular weight, deacetylation $\geq 95 \%$, viscosity 20-300 cP) and sodium tripolyphosphate (TPP) were purchased from Sigma-Aldrich. Curcumin was received as a gift from Krishna Manufacturing (India). Syringe filter, dialysis bag (cutoff molecular weight $12000 \mathrm{Da}$ ), HPLC grade acetonitrile and methanol were purchased from Himedia (Mumbai, India). Eudragit FS 30D was a generous gift of Evonik India Pvt. Ltd, Mumbai. Polyvinyl alcohol (PVA) was purchased from Fisher Scientific India. Milli-Q water used throughout the study was prepared using a Milli-Q 185 purification system (Bedford, Massachusetts). All other reagents and chemicals used were of analytical grade.

\section{Purification of chitosan}

Solid chitosan powder $(1 \mathrm{~g})$ was mixed with $10 \mathrm{ml} 1 \mathrm{M}$ sodium hydroxide $(\mathrm{NaOH})$ solution. This was heated with continuous stirring for $2 \mathrm{~h}$ at $70{ }^{\circ} \mathrm{C}$. The resulting mixture was filtered, and the solid recovered was washed thoroughly with water and dried at $40{ }^{\circ} \mathrm{C}$ for $24 \mathrm{~h}$. The dried flakes were dissolved in $0.1 \mathrm{M}$ acetic acid solution with gentle stirring and insoluble residue removed by filtration. To precipitate chitosan from solution, the $\mathrm{pH}$ value of the filtrate was adjusted to $\mathrm{pH} 8.0$ using $\mathrm{NaOH}$. The precipitated chitosan was washed extensively with purified water and dried under vacuum at $25 \pm 5{ }^{\circ} \mathrm{C}$ for $24 \mathrm{~h} .{ }^{57}$

\section{Preparation of chitosan nanoparticles (CS-NPs)}

CS-NPs were prepared according to the ionotropic gelation method described by Calvo et al. (1997) with slight modifications. ${ }^{58}$ Briefly, CS was dissolved in $1 \%$ aqueous acetic acid solution ( $1 \mathrm{mg} \mathrm{ml}^{-1}$ ) by magnetic stirring until a transparent solution was obtained. Sodium hydroxide was used to adjust the $\mathrm{pH}$ of the solution up to 4.4 to 4.5 and the solution was filtered to remove insoluble particles. TPP was dissolved in double distilled water at a concentration of $1 \mathrm{mg} \mathrm{ml}^{-1}$. Curcumin solution ( $3 \mathrm{ml}, 10 \mathrm{mg} \mathrm{ml}^{-1}$ in ethanol) was added to the TPP solution. Finally, the drug-containing TPP solution was added dropwise to the chitosan solution using a syringe, at the rate of 1 $\mathrm{ml} \min ^{-1}$ under constant stirring at room temperature for $2 \mathrm{~h}$, and the mixture was sonicated. The dispersion formed was centrifuged at $14000 \mathrm{rpm}$ at $4{ }^{\circ} \mathrm{C}$ for $45 \mathrm{~min}$. The supernatant was taken for drug content study, and the sediment was dialysed with distilled water to remove free drug; the dialysed formulation was lyophilized and used for further characterization. 


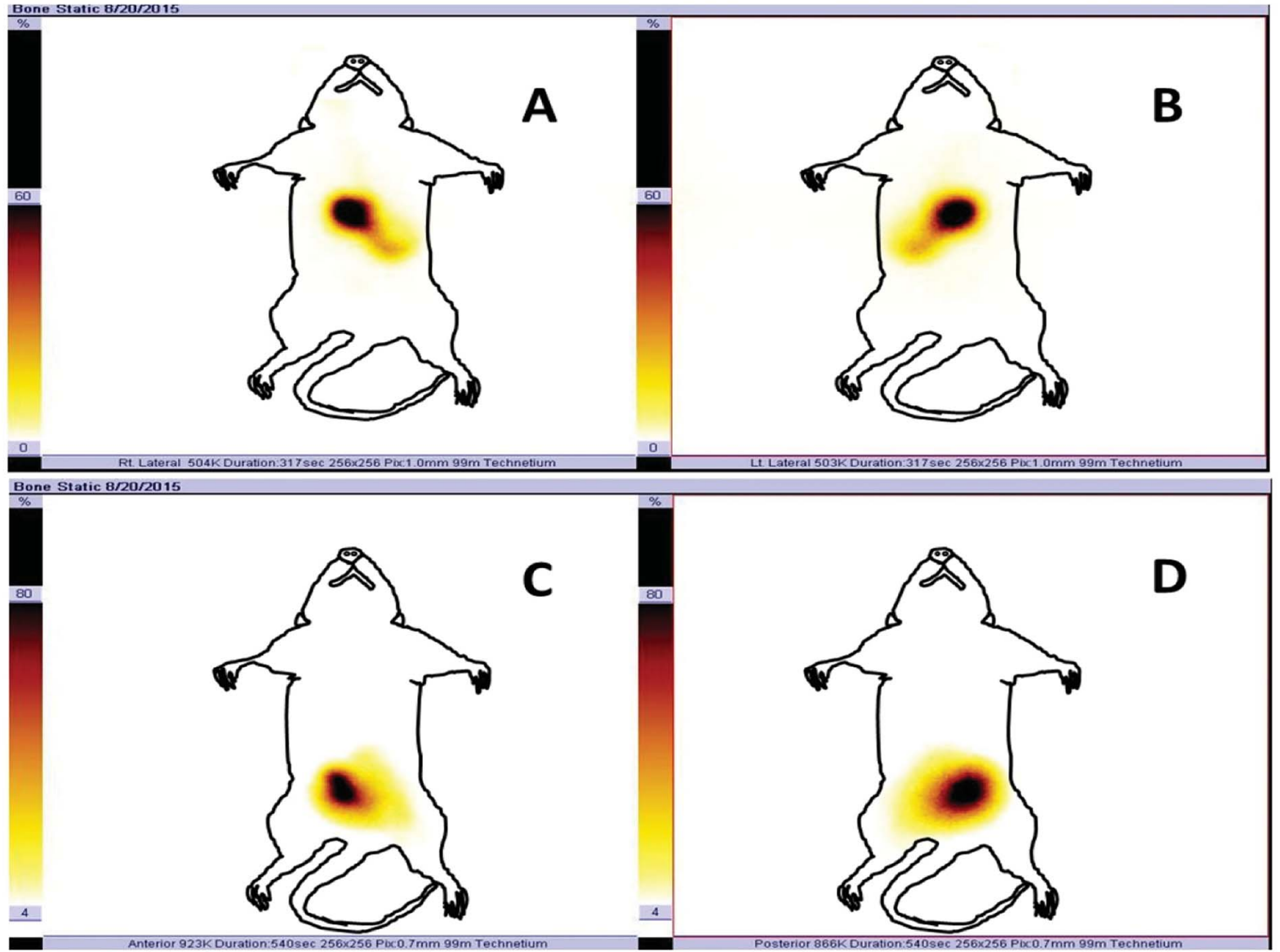

Fig. 9 In vivo gamma-scintigraphic images acquired after oral administration of radiolabelled formulation: (A) and (B) depict the anterior and posterior images, respectively, $3 \mathrm{~h}$ after administration of radiolabelled carrier, while (C) and (D) show anterior and posterior images at $8 \mathrm{~h}$. For clearness, a sketch of the rat is drawn over the images.

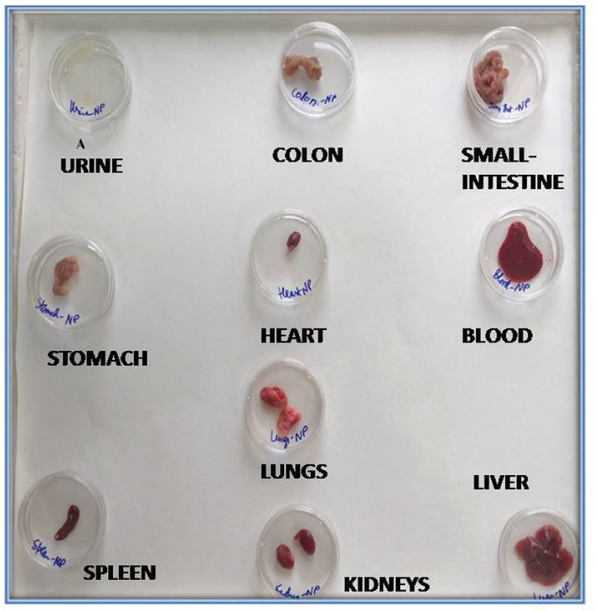

(A)

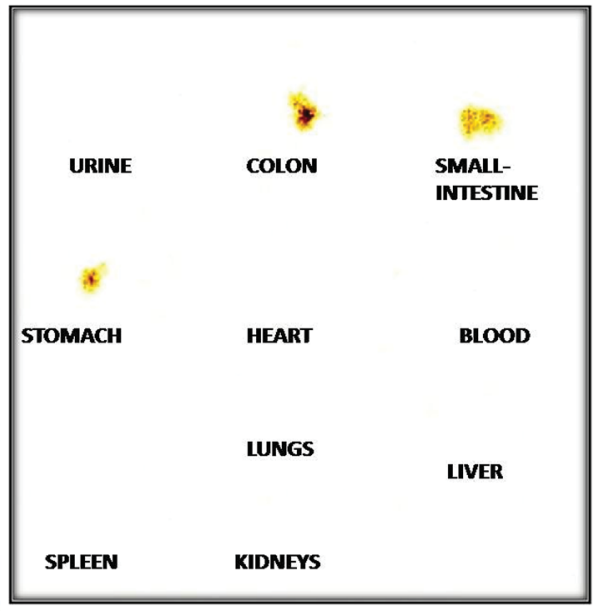

(B)

Fig. 10 Biodistribution study: (A) normal representation, and (B) gamma-scintigraphic image of different isolated organs $24 \mathrm{~h}$ post administration of the radiolabelled formulation in rats. 


\section{Eudragit FS 30D coating on CS-NPs}

The prepared NPs were coated with Eudragit FS 30D by means of an oil-in-oil emulsification solvent evaporation technique using a core:coat ratio $1: 2$. One millilitre of the dispersion of Eudragit FS 30D was dissolved in a mixture of dichloromethane:ethanol:isopropyl alcohol (6 : $5: 4)$. CS-CN-NPs were dispersed in Eudragit FS 30D solution with continuous stirring for $15 \mathrm{~min}$. The obtained dispersion was transferred dropwise into $50 \mathrm{ml}$ light liquid paraffin containing $1 \% \mathrm{w} / \mathrm{v}$ Span 80 with continuous magnetic stirring at $2000 \mathrm{rpm}$ at room temperature for $3 \mathrm{~h}$, after which the organic phase was removed under vacuum. The FS-CS-CN-NPs were collected by centrifugation at $14000 \mathrm{rpm}$ for $30 \mathrm{~min}$, and the sediment was washed extensively with petroleum ether and $n$-hexane to remove liquid paraffin, then lyophilized. FS-CS-NPs without CN were also prepared by the same method. ${ }^{59}$

\section{Particle size, PDI and zeta potential determination}

The average particle size and polydispersity index of CS-NPS were determined in triplicate by photon correlation spectroscopy using a Malvern Zetasizer NanoZS (Malvern 3000HS, France). The zeta potential value of CS-NPs was obtained after adjusting the conductivity to $50 \mu \mathrm{S} \mathrm{cm} \mathrm{cm}^{-1}$ with $0.9 \%$ sodium chloride at $25{ }^{\circ} \mathrm{C}$. Mean values were calculated from analysis of three batches. ${ }^{60}$

\section{Morphological evaluation of nanoparticles}

High-resolution transmission electron microscopy (HRTEM). The structural characteristics of NPs were examined using a high-resolution transmission electron microscope (JEM1400, Jeol, Japan). A drop of suspension was placed on a carbon film-covered copper grid (200 mesh), negatively stained with phosphotungstic acid $(1 \% \mathrm{w} / \mathrm{v})$, then air dried and observed by HRTEM.

Scanning electron microscopy (SEM). The prepared NPs were characterized by shape and surface morphology using a JEOL scanning electron microscope (JSM, 6490 LV). The NPs were mounted on an aluminium stub with carbon-glue and coated using a gold sputter module in a high vacuum evaporator. The photographs were taken at an excitation voltage of $10 \mathrm{kV}$. The magnification was sufficient to depict the detailed morphology of the samples under study. ${ }^{\mathbf{6 1}}$

\section{Fourier-transform infrared spectroscopy (FTIR)}

Various types of bonds present in the samples were detected through FTIR (IR Affinity 1 Schimadzu). FTIR spectra of chitosan, curcumin and CS-NPs (blank and loaded with curcumin) were obtained by mixing $1 \mathrm{mg}$ powder samples with $200 \mathrm{mg}$ of purified and desiccated $\mathrm{KBr}$ to obtain pellets and scanned from 400 to $4000 \mathrm{~cm}^{-1}$ at a resolution of $4 \mathrm{~cm}^{-1}$. The background measurement was performed on a pellet holder with a pellet of $\mathrm{KBr}$ without a sample.

\section{Percentage entrapment efficiency (\%EE)}

To determine encapsulation efficiency of the nanoparticles, an indirect method was adopted. CS-NPs and un-entrapped CN were separated by centrifugation at $18000 \mathrm{rpm}$ using a cooled centrifuge (REMI, India) at $4{ }^{\circ} \mathrm{C}$ for $10 \mathrm{~min}$. The sediment of NPs was further washed with distilled water by centrifugation at the same rpm for $5 \mathrm{~min}$. The amounts of un-entrapped $\mathrm{CN}$ in the first and second supernatants were estimated by means of HPLC (YL 9100, Yunglin, South Korea) with detection at $425 \mathrm{~nm}$. The \%EE was calculated as follows.

$$
\% \mathrm{EE}=\frac{\text { Total drug added }- \text { un-entrapped drug }}{\text { Total drug added }} \times 100
$$

\section{HPLC analysis of curcumin}

An HPLC method was developed and validated for estimating CN. HPLC separation was achieved on a C-18, Cosmosil-packed column (5 $\mu \mathrm{m}$ particle size, $4.6 \mathrm{~mm}$ (ID) $\times 250 \mathrm{~mm}$, Nacalai). Column effluents were monitored at $425 \mathrm{~nm}$. The mobile phase was a mixture of methanol:acetonitrile:phosphate buffer $(42.5: 42.5: 15 . \mathrm{v} / \mathrm{v} / \mathrm{v})$. The $\mathrm{pH}$ of the mobile phase was adjusted to 5 with orthophosphoric acid. The solution was filtered and degassed via a bath sonicator. Chromatography was performed at $25{ }^{\circ} \mathrm{C}$, at a flow rate of $1.0 \mathrm{ml} \mathrm{min}{ }^{-1}$. The calibration curve of $\mathrm{CN}$ was linear in the range of $5-50 \mu \mathrm{g} \mathrm{ml}^{-1}$. The retention time of $\mathrm{CN}$ was found to be $4.5 \mathrm{~min}$.

\section{In vitro swelling}

The swelling study was carried out according to the method reported previously ${ }^{62}$ with some modifications, by placing $100 \mathrm{mg}$ of CS, CS-NPs, CS-CN-NPs and FS-CS-CN-NPs in preweighed centrifuge tubes containing phosphate buffer $(\mathrm{pH}$ 1.2, 6.8 and 7.4). Then NPs were allowed to swell for a period of $0,2,4,6,8,12$ and $24 \mathrm{~h}$ at room temperature. Samples were centrifuged at $10000 \mathrm{rpm}$ for $45 \mathrm{~min}$ at $27^{\circ} \mathrm{C}$. The changes in wet weight were measured after removal of the supernatant, when the weight of the tubes was measured again. The degree of swelling $(\alpha)$ was determined using the following equation:

$$
\alpha=\frac{\left(W_{\mathrm{g}}-W_{0}\right)}{W_{0}}
$$

where $W_{0}$ is the initial weight of CS-NPs and FS-CS-NPs, and $W_{\mathrm{g}}$ is the weight of CS-NPs and FS-CS-NPs at equilibrium swelling in the medium.

\section{Mucoadhesive ex vivo test}

The in vitro interaction of the developed NPs with mucin was assessed according to a previously reported method, with slight modification. ${ }^{63}$ One millilitre NPs suspension was mixed with the same volume of porcine stomach mucin (PSM) type III (Himedia) suspension (0.04\%) in PBS (0.05 M, pH 7.4). After incubation at $37 \pm 1{ }^{\circ} \mathrm{C}$ for $30,60,90,120$ and $180 \mathrm{~min}$, the 
samples were centrifuged at $18000 \mathrm{rpm}$ for $30 \mathrm{~min}$, and the concentration of PSM in the supernatant was examined by UV spectrometry (UV-1800 spectrophotometer, Shimadzu) at $251 \mathrm{~nm}$. The PSM binding efficiency of NPs was estimated from the following equation:

$$
\text { PSM binding efficiency } \%=\frac{\left(C_{0}-C_{\mathrm{s}}\right)}{C_{0}} \times 100
$$

where $C_{0}$ is the initial concentration of PSM used for incubation and $C_{\mathrm{s}}$ is the concentration of free PSM in the supernatant.

\section{In vitro drug release}

An in vitro drug release study of CN, CS-CN-NPs and FS-CS-CNNPs was performed in simulated gastrointestinal fluids, i.e. (a) in simulated gastric fluid (SGF) at pH $1.2(0-2 \mathrm{~h})$, (b) in SGF plus simulated intestinal fluid (SIF) at pH 4.5 (2-4 h), (c) in SIF at pH $6.8(4-6 \mathrm{~h})$ and (d) in simulated colonic fluid (SCF), with or without rat caecal contents, at $\mathrm{pH} 7.4(6-24 \mathrm{~h})$. SCF was prepared by a method reported earlier, with slight modifications. ${ }^{64}$

Pre-treated dialysis bags containing CS-CN-NPs and FS-CSCN-NPs were immersed separately in the above-mentioned dissolution media $(100 \mathrm{ml})$ in sequence for $24 \mathrm{~h}$ at $100 \mathrm{rpm}$ with magnetic stirring at $37 \pm 0.5{ }^{\circ} \mathrm{C}$. Tween $80(1 \% \mathrm{v} / \mathrm{v})$ was added to the dissolution media to facilitate the solubilization of $\mathrm{CN}$ released from formulations. Samples $(2 \mathrm{ml})$ were withdrawn at a predetermined time interval $(0.5,1,2,3,4,5,6,8,12$, and 24 $\mathrm{h}$ ), and replaced with the same volume of respective dissolution medium after each sampling to maintain the sink condition. The samples were centrifuged at $10000 \mathrm{rpm}$ for $15 \mathrm{~min}$, the supernatant was filtered through $0.22 \mu \mathrm{m}$ membrane filter, and the filtrate was subjected to HPLC analysis after appropriate dilution.

Preparation of rat caecal and colonic medium. Male Wistar rats (6-8 weeks old, 120-150 g) were maintained under standard laboratory conditions of $12 \mathrm{~h}$ light and $12 \mathrm{~h}$ dark cycles at $25 \pm$ $2{ }^{\circ} \mathrm{C}$ with pellet diet and water ad libitum and divided into two groups, each group containing 3 animals. All animal experiments were approved by the Institutional Animal Ethical Committee of Guru Ghasidas University Bilaspur and the Committee for the Purpose of Control and Supervision of Experiments on Animals (CPSCEA), Government of India. The simulated colonic fluid was prepared by an enzyme induction method to evaluate the biodegradability of chitosan by colonic bacteria and rate of drug release in the colonic medium. For stimulation of enzymes that particularly act on chitosan during its passage through the colon, $1 \mathrm{ml}$ of $2 \%$ chitosan dispersion in triple distilled water was directly administered to the rat by oral gavage for 7 days before commencement of the experiment to induce these enzymes and provide a good condition for assessing the susceptibility of chitosan to colonic bacterial degradation. ${ }^{65}$ The rats were humanely killed 30 min before starting the study. The abdomen was opened, the caecum was isolated, ligated at both ends, dissected and immediately transferred into simulated intestinal fluid previously bubbled with nitrogen to remove oxygen. The caecal bags were opened; contents were weighed individually, pooled and then suspended in the phosphate buffered saline $\mathrm{pH} 7.4$ to produce a final caecal concentration of $5 \% \mathrm{w} / \mathrm{v}$.

\section{Determination of release kinetics}

The use of in vitro drug dissolution to predict in vivo bioperformance can be considered as the rational development of controlled release formulations, since qualitative and quantitative changes in a formulation may alter drug release and in vivo performance. So, we determined release kinetic models which described the overall release of drug from the developed formulation. We used model-dependent methods (zero order, first order, Higuchi, Korsmeyer-Peppas models) to predict drug release mechanism from CS-CN-NPs and FS-CS-CN-NPs, and the best model was selected by means of correlation coefficient. ${ }^{66}$

\section{In vivo study}

Radiolabelling of FS-CS-NPs with technetium-99m $\left({ }^{99 m_{T c}}\right.$ ). FS-CS-NPs were labelled with ${ }^{99 \mathrm{~m}} \mathrm{Tc}$ by a method reported previously, with some modifications. ${ }^{67}$ Stannous chloride dihydrate $\left(\mathrm{SnCl}_{2} \cdot 2 \mathrm{H}_{2} \mathrm{O}\right)$ was used as a reducing agent. Briefly, the required amount of sterile Tc-99m-pertechnetate (3-4 mCi) was placed in a sterile glass vial followed by addition of 20-200 $\mu \mathrm{g}$ stannous chloride at $\mathrm{pH}$ 6.5-7.0 (adjusted with $0.5 \mathrm{M}$ $\mathrm{NaHCO}_{3}$ ), then the mixture was filtered through a $0.22 \mu \mathrm{m}$ membrane filter. Two millilitre dispersion of a developed formulation was added to the above mixture with continuous mixing. The resultant mixture was incubated at $25 \pm 5{ }^{\circ} \mathrm{C}$ for $30 \mathrm{~min}$.

Radiolabelling efficiency. The radiolabelling efficiency was determined by ascending instant thin-layer chromatography (ITLC). Briefly, an ITLC silica gel strip was used as stationary phase and $100 \%$ acetone as mobile phase. The radiolabelled sample was placed on the chromatographic plate, which was put in the TLC chamber and developed with $100 \%$ acetone. The strip was removed after the solvent reached the top, then dried and cut into different segments, and radioactivity was determined using a gamma counter (CAPRAC-R, CII; Capintech, USA). The labelling efficiency was influenced by the concentration of reducing agent, $\mathrm{pH}$ and incubation time. ${ }^{68}$ The stability of the radiolabelled formulation was evaluated in $0.9 \%$ $(\mathrm{w} / \mathrm{v})$ sodium chloride and rat plasma as reported by Jain et al. ${ }^{69}$

In vivo scintigraphic imaging. Gamma $(\gamma)$-scintigraphy was used to monitor the passage of orally administered NPs and assess the release pattern of drug qualitatively throughout the GIT. Sprague Dawley (male) rats weighing $190 \pm 20 \mathrm{~g}$ were used for $\gamma$-scintigraphic imaging. Animals were procured from the animal house of INMAS (New Delhi). The rats were fasted overnight. Each animal received a single dose of radiolabelled NPs (3-4 mCi, $20 \mathrm{mg}$ ) by oral gavage. Animals were then anaesthetized by intramuscular injection of ketamine hydrochloride (100 $\left.\mathrm{mg} \mathrm{kg}^{-1}\right)$ and xylazine $\left(10 \mathrm{mg} \mathrm{kg}^{-1}\right) 10 \mathrm{~min}$ before imaging. The animals were fixed on a board in the posterior and anterior positions, and imaging was performed using a planar 
gamma camera (GE Hawkeye $囚$ ) at $3 \mathrm{~h}$ and $8 \mathrm{~h}$ post administration.

Biodistribution studies. Sprague Dawley (male) rats weighing $190 \pm 20 \mathrm{~g}$ were selected for the study. The rats were fasted overnight, and each animal received a single dose of radiolabelled NPs by the oral route. The rats were killed humanely after $24 \mathrm{~h}$ administration of NPs. The blood was collected by cardiac puncture, and then lungs, heart, liver, spleen, kidney, stomach, small intestine, large intestine and urine were collected. All the organs were washed with normal saline and put into transparent glass Petri dishes for gamma imaging.

\section{Statistical analysis}

The results are expressed as mean values \pm standard deviation (SD). The statistical analysis was done by using Student's $t$-test and one-way analysis of variance (ANOVA). A probability level of $P<0.05$ was considered to be statistically significant.

\section{Conclusions}

Chitosan nanoparticles prepared by an ionic gelation method were nano-sized and uniformly dispersed. FTIR spectra of the formulations confirmed the cross-linking of CS with TPP. $\mathrm{pH}$ stimulated delivery of $\mathrm{CN}$ to colon was successfully attained via coating of CS-NPs with Eudragit FS 30D. In vitro drug release studies confirmed controlled release in the colon, which also correlated with gamma-scintigraphy. The morphological analysis showed CS-NPs have a slightly rough surface and FS-CS-NPS having a smooth surface. The release mechanism of the drug has super case II diffusion kinetics, indicating swelling and relaxation of the polymer. The prepared formulation of $\mathrm{CN}$ employing $\mathrm{pH}$ stimulated delivery provides a potential approach to treat ulcerative colitis, and the system can also be used for targeting other drugs to the colon.

\section{Conflicts of interest}

There are no conflicts to declare.

\section{Acknowledgements}

One of the authors, Pooja Mongia, very gratefully acknowledges UGC, New Delhi for providing financial assistance to RGNFSRF. The authors are also grateful to the electron microscopic division of AIIMS, New Delhi and USIC of Babasaheb Bhimrao Ambedkar University Lucknow for use of their TEM and SEM facilities respectively.

\section{References}

1 T. Bautzova, M. Rabiskova and A. Lamprecht, Drug Dev. Ind. Pharm., 2011, 37, 1100-1109.

2 A. B. Pithadia and S. Jain, Pharmacol. Rep., 2011, 63, 629-642. 3 B. A. G. Hendrickson, R. Gokhale and J. H. Cho, Clin. Microbiol. Rev., 2002, 15, 79-94.

4 K. Conrad, D. Roggenbuck and M. W. Laass, Autoimmun. Rev., 2014, 13, 463-466.
5 A. Kaser, S. Zeissig and R. S. Blumberg, Annu. Rev. Immunol., 2010, 28, 573-621.

6 D. L. Suskind, G. Wahbeh, T. Burpee, M. Cohen, D. Christie and W. Weber, J. Pediatr. Gastroenterol. Nutr., 2013, 56, 277279.

7 O. H. Nielsen, Scand. J. Gastroenterol., 1982, 17, 389-393.

8 S. I. Grivennikov, Semin. Immunopathol., 2013, 35, 229-244.

9 L. Hartnett and L. J. Egan, Carcinogenesis, 2012, 33, 723-731.

10 A. Beloqui, R. Coco, P. B. Memvanga, B. Ucakar, A. des Rieux and V. Préat, Int. J. Pharm., 2014, 473, 203-212.

11 C. Lahiff and A. C. Moss, Inflamm. Bowel Dis., 2011, 17, E66.

12 M. S. Baliga, N. Joseph, M. V. Venkataranganna, A. Saxena, V. Ponemone and R. Fayad, Food Funct., 2012, 3, 1109-1117.

13 W. B. Song, Y. Y. Wang, F. S. Meng, Q. H. Zhang, J. Y. Zeng, L. P. Xiao, X. P. Yu, D. D. Peng, L. Su, B. Xiao and Z. S. Zhang, PLoS One, 2010, 5, e12969.

14 A. Goel, A. B. Kunnumakkara and B. B. Aggarwal, Biochem. Pharmacol., 2008, 75, 787-809.

15 M. V. Venkataranganna, M. Rafiq, S. Gopumadhavan, G. Peer, U. V. Babu and S. K. Mitra, World J. Gastroenterol., 2007, 13, 1103-1107.

16 S. C. Gupta, S. Patchva and B. B. Aggarwal, AAPS J., 2013, 15, 195-218.

17 N. Adapala and M. M. Chan, Lab. Invest., 2008, 88, 13291339.

18 P. Arya and K. Pathak, Int. J. Pharm., 2014, 460, 1-12.

19 G. R. Irving, A. Karmokar, D. P. Berry, K. Brown and W. P. Steward, Best Pract. Res., Clin. Gastroenterol., 2011, 25, 519-534.

20 P. Dulbecco and V. Savarino, World J. Gastroenterol., 2013, 19, 9256-9270.

21 B. Xiao, X. Si, M. Zhang and D. Merlin, Colloids Surf., B, 2015, 135, 379-385.

22 S. Setthacheewakul, S. Mahattanadul, N. Phadoongsombut, W. Pichayakorn and R. Wiwattanapatapee, Eur. J. Pharm. Biopharm., 2010, 76, 475-485.

23 Z. Song, R. Feng, M. Sun, C. Guo, Y. Gao, L. Li and G. Zhai, J. Colloid Interface Sci., 2011, 354, 116-123.

24 P. Jourghanian, S. Ghaffari, M. Ardjmand, S. Haghighat and M. Mohammadnejad, Adv. Pharm. Bull., 2016, 6, 17-21.

25 H. Ji, J. Tang, M. Li, J. Ren, N. Zheng and L. Wu, Drug Delivery, 2016, 23, 459-470.

26 N. Madusanka, K. M. de Silva and G. Amaratunga, Carbohydr. Polym., 2015, 134, 695-699.

27 A. Lamprecht, N. Ubrich, H. Yamamoto, U. Schafer, H. Takeuchi, P. Maincent, Y. Kawashima and C. M. Lehr, J. Pharmacol. Exp. Ther., 2001, 299, 775-781.

28 P. K. Mongia, R. Khatik, R. Raj, N. Jain and A. K. Pathak, J. Biomater. Tissue Eng., 2014, 4, 738-743.

29 V. R. Sinha, A. K. Singla, S. Wadhawan, R. Kaushik, R. Kumria, K. Bansal and S. Dhawan, Int. J. Pharm., 2004, 274, 1-33.

30 J. W. Lee, J. H. Park and J. R. Robinson, Int. J. Pharm., 2010, 89, 850-866.

31 S. A. Pathan, Z. Iqbal, J. K. Sahani, S. Talegaonkar, R. K. Khar and F. J. Ahmad, Recent Pat. Drug Delivery Formulation, 2008, 2, 177-188. 
32 G. P. Andrews, T. P. Laverty and D. S. Jones, Eur. J. Pharm. Biopharm., 2009, 71, 505-518.

33 D. El-Nashar, N. Saleh, A. Soliman and F. Helaly, Study the release kinetics of curcumin released from PVA/curcumin composites and its evaluation towards hepatocarcinoma, $J$. Appl. Pharm. Sci., 2016, 6, 067-072.

34 P. Verderio, P. Bonetti, M. Colombo, L. Pandolfi and D. Prosperi, Biomacromolecules, 2013, 14, 672-682.

35 M. M. Yallapu, M. Jaggi and S. C. Chauhan, Drug discovery today, 2012, 17, 71-80.

36 C. Gao, F. Tang, G. Gong, J. Zhang, M. P. M. Hoi, S. M. Y. Lee and R. Wang, Nanoscale, 2017, 9, 12533-12542.

37 L. H. Chuah, N. Billa, C. J. Roberts, J. C. Burley and S. Manickam, Pharm. Dev. Technol., 2013, 18, 591-599.

38 F. Akhtar, M. M. Rizvi and S. K. Kar, Biotechnol. Adv., 2012, 30, 310-320.

39 L. H. Chuah, C. J. Roberts, N. Billa, S. Abdullah and R. Rosli, Colloids Surf., B, 2014, 116, 228-236.

40 K. P. Seremeta, D. A. Chiappetta and A. Sosnik, Colloids Surf., $B, 2013,102,441-449$.

41 A. Makhlof, Y. Tozuka and H. Takeuchi, Eur. J. Pharm. Biopharm., 2009, 72, 1-8.

42 T. F. Vandamme, A. Lenourry, C. Charrueau and J. C. Chaumeil, Carbohydr. Polym., 2002, 48, 219-231.

43 L. Zimova, D. Vetchy, J. Muselik and J. Stembirek, Drug Delivery, 2012, 19, 81-89.

44 V. K. Gupta, T. E. Beckert and J. C. Price, Int. J. Pharm., 2001, 213, 83-91.

45 R. A. Taylor and M. C. Leonard, Altern Med Rev, 2011, 16, 152-156.

46 M. Prabaharan and J. F. Mano, Drug Delivery, 2004, 12, 4157.

47 A. Anitha, V. G. Deepagan, V. V. Divya Rani, D. Menon, S. V. Nair and R. Jayakumar, Carbohydr. Polym., 2011, 84, 1158-1164.

48 J. S. Choi, Int. J. Biol. Macromol., 2016, 92, 850-859.

49 R. K. Das, N. Kasoju and U. Bora, Nanomedicine: Nanotechnology, Biology and Medicine, 2010, 6, 153-160.

50 A. Anitha, N. Deepa, K. P. Chennazhi, V. K. Lakshmanan and R. Jayakumar, Biochim. Biophys. Acta, 2014, 1840, 2730-2743.
51 P. Udompornmongkol and B. H. Chiang, J. Biomater. Appl., 2015, 30, 537-546.

52 S. M. Lim, D. K. Song, S. H. Oh, D. S. Lee-Yoon, E. H. Bae and J. H. Lee, J. Biomater. Sci., Polym. Ed., 2008, 19, 453-466.

53 M. Sampath, R. Lakra, P. Korrapati and B. Sengottuvelan, Colloids Surf., B, 2014, 117, 128-134.

54 S. A. Mortazavi, Int. J. Pharm., 1995, 124, 173-182.

55 I. Aguilar-Rosas, S. Alcala-Alcala, V. Llera-Rojas and A. Ganem-Rondero, Drug Dev. Ind. Pharm., 2015, 41, 16321639.

56 S. Papadimitriou, D. Bikiaris, K. Avgoustakis, E. Karavas and M. Georgarakis, Carbohydr. Polym., 2008, 73, 44-54.

57 Q. Gan and T. Wang, Colloids Surf., B, 2007, 59, 24-34.

58 P. Calvo, C. Remuñán-López, J. L. Vila-Jato and M. J. Alonso, J. Appl. Polym. Sci., 1997, 63, 125-132.

59 T. Oosegi, H. Onishi and Y. Machida, Eur. J. Pharm. Biopharm., 2008, 68, 260-266.

60 W. Fan, W. Yan, Z. Xu and H. Ni, Colloids Surf., B, 2012, 90, 21-27.

61 R. Raj, P. M. Raj and A. Ram, RSC Adv., 2016, 6, 53578-53586.

62 A. R. Dudhani and S. L. Kosaraju, Carbohydr. Polym., 2010, 81, 243-251.

63 Y. Yin, D. Chen, M. Qiao, Z. Lu and H. Hu, J. Controlled Release, 2006, 116, 337-345.

64 N. Bashardoust, J. L. Jenita and P. Zakeri-Milani, Adv. Pharm. Bull., 2011, 1, 87-92.

65 Y. S. Krishnaiah, A. Seetha Devi, L. Nageswara Rao, P. R. Bhaskar Reddy, R. S. Karthikeyan and V. Satyanarayana, J. Pharm. Pharm. Sci., 2001, 4, 235-243.

66 R. Alpana, M. R. Pooja, K. Nitish and R. Rakesh, Pharm. Nanotechnol., 2016, 4, 316-328.

67 A. Kaul, P. P. Hazari, H. Rawat, B. Singh, T. C. Kalawat, S. Sharma, A. K. Babbar and A. K. Mishra, Int. J. Infect. Dis., 2013, 17, e263-270.

68 S. Katiyar, J. Pandit, R. S. Mondal, A. K. Mishra, K. Chuttani, M. Aqil, A. Ali and Y. Sultana, Carbohydr. Polym., 2014, 102, 117-124.

69 V. Jain, N. K. Swarnakar, P. R. Mishra, A. Verma, A. Kaul, A. K. Mishra and N. K. Jain, Biomaterials, 2012, 33, 72067220 . 